\title{
Versatile Optochemical Quantification with Optical Mouse
}

\author{
Altamash Fakki, ${ }^{1}$ Salahaldein Ahmed, ${ }^{1}$ Jongwon Park, ${ }^{2}$ and Chang-Soo Kim ${ }^{1,3}$ \\ ${ }^{1}$ Electrical \& Computer Engineering, Missouri University of Science and Technology, 401 W. 16th St., Rolla, MO 65409, USA \\ ${ }^{2}$ Department of High-Tech Medical System, Kyungil University, 50 Gamasil-gil, Gyeongsan, Gyeongsangbuk-do, Republic of Korea \\ ${ }^{3}$ Chemical \& Biochemical Engineering, Missouri University of Science and Technology, 401 W. 16th St., Rolla, MO 65409, USA
}

Correspondence should be addressed to Chang-Soo Kim; ckim@mst.edu

Received 19 March 2017; Revised 19 May 2017; Accepted 24 May 2017; Published 6 August 2017

Academic Editor: Jesus Corres

Copyright (C) 2017 Altamash Fakki et al. This is an open access article distributed under the Creative Commons Attribution License, which permits unrestricted use, distribution, and reproduction in any medium, provided the original work is properly cited.

\begin{abstract}
There is an ever increasing need for simple, low-cost instruments for ubiquitous medical and environmental measurements in conjunction with networks and Internet-of-things. This work demonstrates that the optical mouse, one of the most common optoelectronic computer peripherals, can be used for chemical quantification. Particularly, we explore the feasibility of using the preassembled optical platform of mouse for oxygen and $\mathrm{pH}$ quantification. The image sensor and the light-emitting diode (LED) serve as photodetector and excitation/illumination light source, respectively, while the preinstalled microoptics (e.g., lens and waveguide) provide a fixed optical arrangement convenient for sample analysis. This novel, cost-effective approach demonstrates the potential application of optical mouse for bioanalytical devices in conjunction with commercial sensor strips or simple microfluidic elements. This is one viable option for seamless integration of bioanalytical capability into existing personal computers and associate networks without significant additional hardware.
\end{abstract}

\section{Introduction}

Optical mouse is navigating device to display the location of cursors on the computer screens. The light source inside the optical mouse illuminates the surface on which the mouse moves. This allows the image sensor to monitor the reflected microscopic surface images continuously as the mouse changes its location and calculates the displacement. The preinstalled microoptics determines the certain illumination angle of light source and the focal length of imager. Many examples adopting the mouse for other applications than this original purpose are still mostly motion sensing to obtain mechanical parameters [1-4].

Optical sensing in medical diagnosis and chemical analysis becomes an attractive and dominant technology with the advancement of various optoelectronic devices. Particularly, there is an ever increasing need for simple, low-cost instruments for ubiquitous medical and environmental measurements with networking capabilities [5-7]. Since the optical mouse is already equipped with a set of optical components (i.e., photodetector, light source, lens, and waveguide), this device platform is particularly suitable for various optical detection modalities including transmission, reflection, absorption, and emission of light signals. Therefore, this research attempts to explore the feasibility of using an optical mouse as a ubiquitous, low-cost bioanalytical platform by taking advantage of such built-in optical configuration of mouse. Previously, we have presented preliminary chemical measurements with optical mouse for the first time [8]. This report expands this concept to demonstrate the capability of the optical mouse as bioanalytical device for both oxygen and $\mathrm{pH}$ quantification in detail.

Optical oxygen sensors are becoming more common in many applications compared to traditional oxygen electrodes. The optical oxygen sensor works on the principle where oxygen quenches the luminescence emitted from dyes that are immobilized in a porous permeable medium [9]. For the oxygen sensor platform utilizing the mouse, commercial oxygen-sensitive patches are used as the oxygen-sensitive component. A blue LED installed in the optical mouse excites the luminescent dyes immobilized on the oxygen-sensitive 


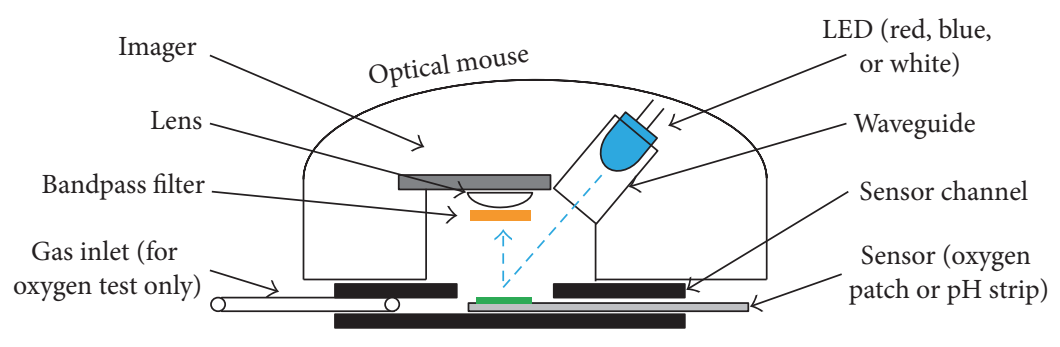

FIGURE 1: Cross-section of the bioanalytical platform based on optical mouse with a fluidic sensor channel to house the commercial sensors. The original red light-emitting diode (LED) is replaced with others (blue or white) for a series of experiments.

patches. The image sensor of the optical mouse is used to register gray scale oxygen patch images with a band pass filter.

Determination of $\mathrm{pH}$ is one of the most frequently required tests in many chemical reactions, biomedical diagnosis, and environment surveillance. The most simple, disposable, and semiquantitative testing for $\mathrm{pH}$ level is to use the $\mathrm{pH}$ test strips incorporating the color-changing $\mathrm{pH}$ indicating dyes [10]. Colorimetric $\mathrm{pH}$ measurements are conducted with the optical mouse utilizing commercial $\mathrm{pH}$ test strips. All images are taken with the image sensor and the influence of factors for image acquisition such as optical filter and light source and gray scale image analysis method are evaluated. Other $\mathrm{pH}$ evaluation approaches using spectrophotometer and digital color camera are also conducted and compared with data obtained with optical mouse.

\section{Experimental}

2.1. Oxygen Test Platform. A commercially available optical mouse (M-BJ69, Logitech) was modified as an optical oxygen sensor platform by adding a simple fluidic sensor channel beneath the optical mouse allowing sample gases to flow. Figure 1 is the diagram describing the arrangement of optical mouse, bandpass filter (BPF), sensor channel, and commercial oxygen sensor patch. The RedEye oxygen sensor patch (FOXY, $8 \mathrm{~mm}$ diameter, Ocean Optics) is based on ruthenium complex dye used as the oxygen-recognition agent. To excite the dye, the factory-installed red LED inside the optical mouse (630 $\mathrm{nm}$ peak) was replaced by a blue LED (151053BS04500, $465 \mathrm{~nm}$ peak, Wurth Electronics) that can be operated with the same bias condition as that of the original red LED. A $1.1 \mathrm{~mm}$ thick bandpass filter (BPF) $(5 \mathrm{~mm} \times 5 \mathrm{~mm}, 615 \mathrm{~nm}$ peak, Pixelteq) was simply attached at the front of the image sensor lens to allow only the spectral range of interest (i.e., fluorescence emission around $600 \mathrm{~nm}$ ) to increase the oxygen sensitivity.

A sensor channel was prepared using microscope glass slides and adhesives to house the oxygen patch placed beneath the image sensor. An opening of $8 \mathrm{~mm} \times 8 \mathrm{~mm}$ square on the channel was made to expose the oxygen patch for registering images. The setup was covered with a black opaque cloth during the measurement to avoid any external light interference. A mass flow controller (247C 4, MKS) was used to control the ratio of dry oxygen and nitrogen gases from cylinders to formulate the sample gases with different oxygen saturation levels (i.e., $0-100 \%$ ). The prepared sample gas was infused into an embedded polymer tube at the front side of the sensor channel. The sample gas was allowed to flow over the oxygen sensor patch for 15 minutes before registering the images.

2.2. $\mathrm{pH}$ Test Platform. The $\mathrm{pH}$ measurement setup shared the same optical platform as the oxygen case except for two differences. The original red LED (630 nm peak) was used as is to illuminate the $\mathrm{pH}$ test strips. The same sensor channel without the gas tube was used to provide the same measurement condition as that of oxygen tests, although it is not critically required for $\mathrm{pH}$ tests. The same $\mathrm{BPF}$ was used to filter the reflected light from $\mathrm{pH}$ strips. Two different series of experiments were conducted for $\mathrm{pH}$ strip imaging and valuation: (1) red LED source with BPF and (2) white LED source with or without BPF. The purpose of the two series of experiments was to compare the effectiveness of the red and white LED in quantitative $\mathrm{pH}$ valuation.

Laboratory grade plastic $\mathrm{pH}$ test strips (Fisher Scientific) were used for examination. The strip consisted of a cellulose matrix containing dyes which change their colors responding to different $\mathrm{pH}$ levels. Seven $\mathrm{pH}$ buffer solutions (Fisher Scientific) were used for $\mathrm{pH}$ measurements. The expected color changes with respect to buffer solutions were redorange for $\mathrm{pH} 2$, orange for $\mathrm{pH} 4$, yellow for $\mathrm{pH}$ 6, yellowgreen for $\mathrm{pH} 7$, green for $\mathrm{pH} 8$, green-blue for $\mathrm{pH} 10$, and dark blue for $\mathrm{pH} 12$. A $0.016 \mathrm{ml}$ of $\mathrm{pH}$ buffer solution was dropped on a $\mathrm{pH}$ strip and waited for 30 seconds to allow the cellulose matrix to change its color completely before taking images.

2.3. Instrumentation. The image sensor (ADNS 2051, Avago Technologies) inside the mouse used in this study acquired all sensor images. The Arduino-Duemilanove board, a microcontroller interfacing board built onto a single printed circuit board [11], served as the data mediator between the mouse and the computer. It was programmed to fetch the pixel data from the image sensor of the mouse and transmit to the personal computer as depicted in Figure 2.

The computer had a Java application running to receive and display the pixel data coming from the mouse. This application also allowed saving the live images. Registered images from the optical mouse were $16 \times 16$ pixels and each pixel produces 6-bit intensity ranging from zero (i.e., complete black) to 63 (i.e., complete white). The blackand-white image sensors based on the silicon technology image sensor, including the one used in this study, exhibit 


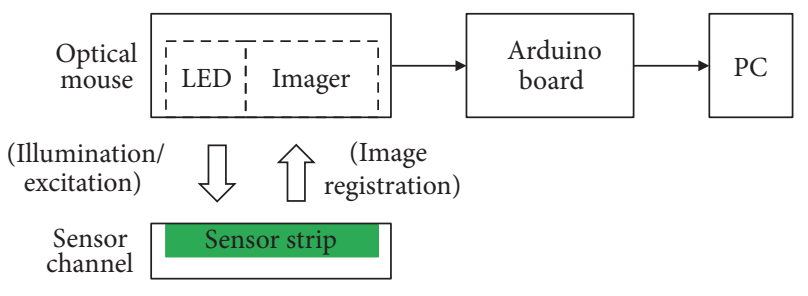

FIGURE 2: Block diagram of instrumentation to interface the image sensor (ADNS 2051) and the personal computer via an ArduinoDuemilanove board.

a responsivity over the visible light range [12]. Therefore, extracting the intensity of target spectral range selectively from the image was important for maximizing the sensitivity of both oxygen and $\mathrm{pH}$. The $\mathrm{BPF}$ enabled the image sensor to register the intensity of target range selectively in gray scale to minimize the contribution from undesired spectral range.

2.4. Image Analysis. The intensities of gray scale data of sensor images were translated into the oxygen saturation levels or $\mathrm{pH}$ values in various samples. In order to reduce the noise, six images were taken continuously to obtain an average value. MATLAB codes were written for processing gray scale images. The process consisted of three steps after image acquisitions: (1) cropping data from the region of interest (ROI) from each image, (2) averaging the intensities of all pixel values of each cropped image, and (3) averaging the intensities of six images.

\section{Results}

3.1. Oxygen Analysis. The most common way to correlate the oxygen saturation level with measured emission intensity is to use the Stern-Volmer equation as follows [13]:

$$
\frac{I_{\mathrm{o}}}{I}=1+K_{\mathrm{sv}}\left[\mathrm{O}_{2}\right]
$$

where $I$ indicates emission intensity in the presence of oxygen (the subscript "o" denotes the absence of oxygen) and $\left[\mathrm{O}_{2}\right]$ and $K_{\mathrm{sv}}$ are oxygen saturation level and Stern-Volmer constant, respectively. Since the emission is quenched by the presence of oxygen, less intensity is expected with increasing oxygen level. This equation produces a linear relationship between the two parameters (intensity ratio $I_{\mathrm{o}} / I$ and oxygen level $\left.\left[\mathrm{O}_{2}\right]\right)$. The average value of all pixel intensities of an image registered at a zero oxygen condition (i.e., $I_{\mathrm{o}}$ ) was divided by that from another image at certain oxygen environment (i.e., $I$ ) to produce the parameter $I_{\mathrm{o}} / I$.

As mentioned in the previous section, the average intensities of the gray scale images were used to estimate the emission intensity responding to oxygen. Figure 3 shows the plot of oxygen sensitivity obtained with the mouse. The oxygen molecule serves as the emission quencher, resulting in weaker intensities as the oxygen saturation level increased. We have an extensive experience on the optical oxygen quantification utilizing the ruthenium complex dyes with both traditional spectrophotometers and rather nontraditional equipment,

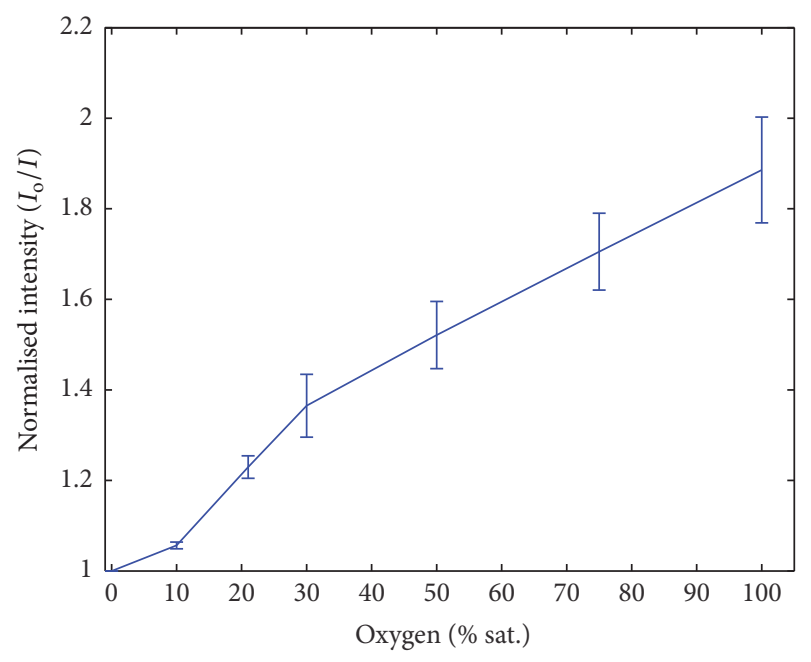

Figure 3: Oxygen sensitivity plots based on the Stern-Volmer equation obtained with optical mouse and blue LED illumination $(n=3)$.

including digital color cameras, blue/white LEDs, and liquid crystal displays (LCD) [13-15]. The sensitivity obtained in this study $\left(I_{\mathrm{o}} / I=1.89\right.$ in $0 \sim 100 \%$ oxygen range $)$ with an optical mouse is in a comparable range with those data in our previous reports $(1.20 \sim 2.42$ in $0 \sim 100 \%$ oxygen $[13,14]$ and 1.31 in $0 \sim 20 \%$ oxygen [15]). Therefore, the optical components in the mouse used in this study exhibit a similar performance to other approaches.

3.2. pH Analysis. Compared to the relatively simple intensity measurement of oxygen (i.e., single frequency), the $\mathrm{pH}$ analysis involved a colorimetric approach (i.e., multiple frequency). In general, disposable $\mathrm{pH}$ test strips are based on the $\mathrm{pH}$-induced irreversible changes in the optical absorbance (thus reflectance) of $\mathrm{pH}$ indicating dyes, which, in turn, produce the overall color variation responding to different $\mathrm{pH}$ samples. In our case, the $\mathrm{pH}$ quantification was mostly associated with the absorption of red wavelengths by the $\mathrm{pH}$ dye in the strip used in this study (not known). Similarly to the oxygen analysis, the grayscale information extracted from the original images of the $\mathrm{pH}$ strips was used to translate into $\mathrm{pH}$ valuation.

First, we replaced the original red LED with a white LED to acquire the $\mathrm{pH}$ strip images. This procedure was conducted because the reflection-based colorimetric analysis normally utilized white light illumination. The silicon photodetector, including ADNS 2051 image sensor used in this study, has a higher responsivity to red wavelengths than other visible ranges [12]. Therefore, the $\mathrm{pH}$ sensitivity was tested with and without the BPF to compare both cases. The sensitivity degradation is expected without BPF as shown in Figure 4(a) because of the interfering wavelengths other than the red range. However, it still exhibits a similar degree of error ranges.

Secondly, another series of measurements were conducted with the preinstalled red LED in the mouse. Figure 4(b) shows the sensitivity of reflected intensities from 


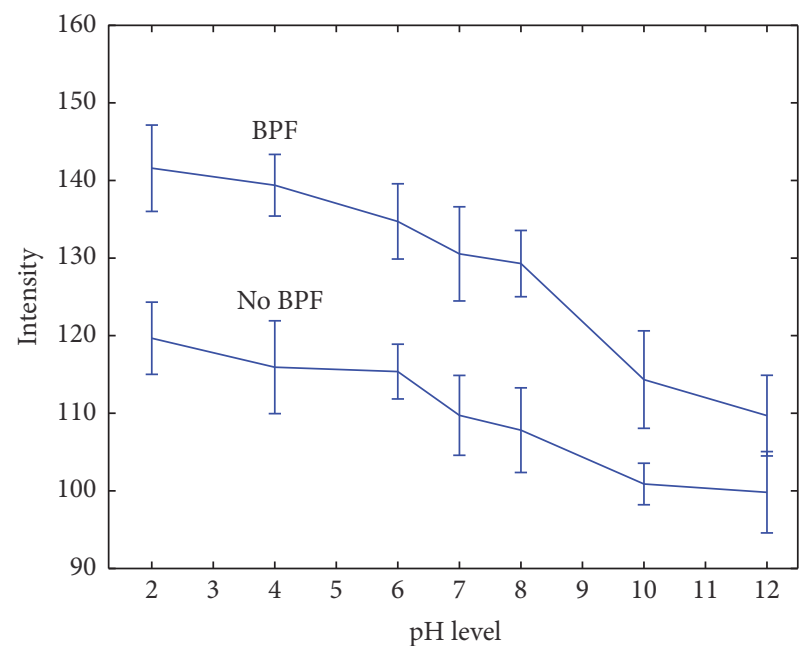

(a)

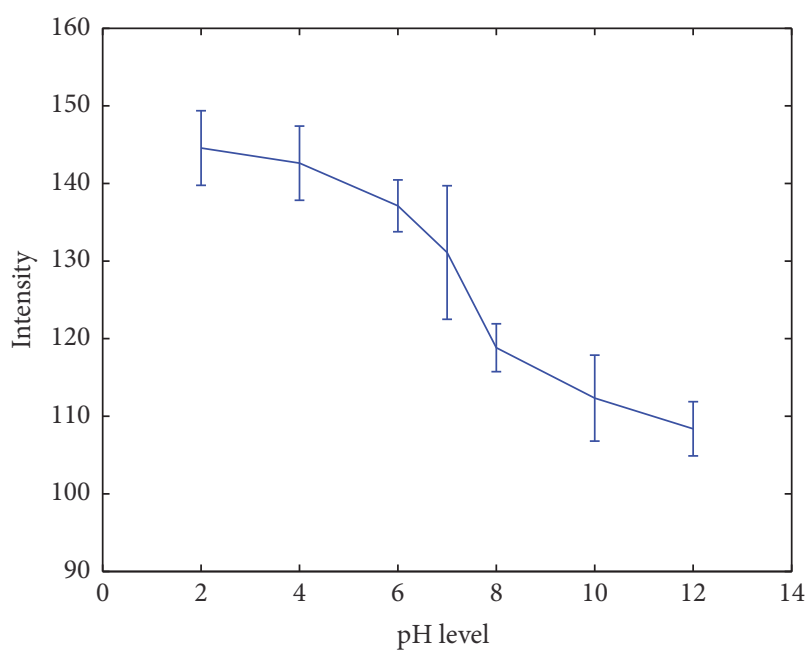

(b)

FIGURE 4: (a) pH sensitivity obtained with optical mouse under white LED illumination with and without the bandpass filter (BPF) to compare the sensitivities $(n=7)$. (b) pH sensitivity obtained under red (LED) illumination with BPF $(n=7)$.

the $\mathrm{pH}$ strips with red light illumination and the use of BPF. About $13 \%$ increase of sensitivity (i.e., the intensity difference between $\mathrm{pH} 2$ and $\mathrm{pH}$ 12) compared with that of the white illumination with BPF is observed. This is because the white LED is a broad band source covering the overall visible spectral range, such that this undesirable range contributed the overall intensity of the silicon image sensor. Therefore, the highest sensitivity can be obtained with the use of red light source in conjunction with BPF to selectively extract the red wavelength range.

3.3. $p H$ Analysis by Other Approaches. In parallel with the $\mathrm{pH}$ quantification using the mouse, the colorimetric change due to absorption of red light was quantified to confirm the results obtained with mouse. A spectrophotometer (USB4000, Ocean Optics) was used in conjunction with a fiber optic probe (R400-7-UV-VIS, Ocean Optics) to analyze the reflectance from the $\mathrm{pH}$ strips under red LED illumination. The probe and the LED were arranged in a similar manner as the optical mouse configuration such that the reflected light can be harvested without direct interference from the LED source. As expected, the red peak intensity decreases as the $\mathrm{pH}$ level increases, which implies that the colorimetric $\mathrm{pH}$ indicating agents absorb the red spectral range increasingly with higher $\mathrm{pH}$. Accordingly, this results in a change from reddish to bluish colors when the sample $\mathrm{pH}$ changes from low to high levels.

A similar intensity profile in Figure 5(b) was obtained while analyzing the red peak level $(630 \mathrm{~nm})$ from the spectra in Figure 5(a). About $12 \%$ increase in sensitivity (i.e., the intensity difference between $\mathrm{pH} 2$ and $\mathrm{pH} 12$ ) with the red LED compared to that with white LED should be noted. This increment is in good agreement with that of $13 \%$ increment obtained with the mouse (i.e., comparison between Figures 4(a) and 4(b) both with BPF). This confirms that the optical mouse is an effective alternative to traditional instruments.
A color CCD camera (DS-5M, Nikon) was used for color image acquisition of $\mathrm{pH}$ strips for analyzing the intensities of primary colors: red, green, and blue (RGB). The color images were registered under white LED illumination. The color image sensors are equipped with the on-chip BPF array (i.e., Bayer filter) that has three spectral ranges for primary colors (i.e., RGB) to separate the visible wavelength range into three colors [12]. Therefore it can be considered as a primitive spectrophotometer with a limited capability.

Figure 6(a) displays the original color images of $\mathrm{pH}$ strips. The cropped color images were analyzed in RGB color space with a MATLAB script to analyze the contribution of each primary color to the images. The red color curve in Figure 6(b) exhibits a similar fashion of decreasing intensity with increasing $\mathrm{pH}$ level, which is in good agreement with other approaches. The red color exhibited the highest sensitivity (i.e., the intensity difference between $\mathrm{pH} 2$ and $\mathrm{pH} 12$ ) among the others. This plot justifies the advantage of using the red LED for illumination for the $\mathrm{pH}$ analysis with mouse (Figure 4).

\section{Discussion}

Overall, as previously stated, the observed responses to oxygen and $\mathrm{pH}$ are in good agreement with those obtained with other instruments as in our previous studies [13-15] for oxygen and in Figures 5 and 6 for $\mathrm{pH}$, respectively. Nevertheless, the image sensor utilized in this study has a limited capability that is intended only for the original purpose of tracking function. This image sensor generates only 64-level (i.e., 6-bit) digitized intensity with respect to light intensity. This fact results in the limited intensity resolution and larger error range compared to those obtained with spectrometer and color camera. Therefore, image sensors with a better intensity resolution may be adopted for advanced applications where a more accurate measurement 


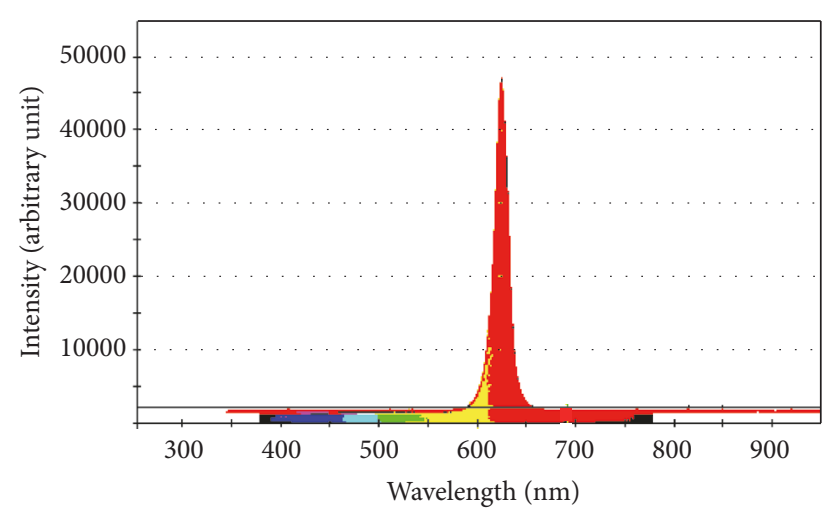

(a)

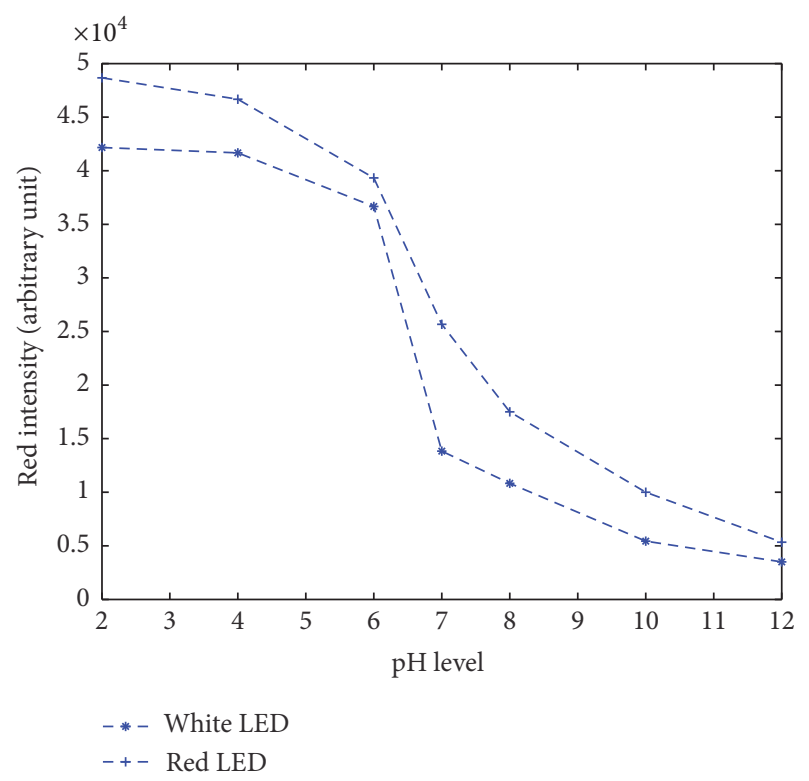

(b)

FIGURE 5: $\mathrm{pH}$ analysis with a spectrophotometer. (a) Spectra of reflectance from a $\mathrm{pH}$ test strip (applied with a $\mathrm{pH} 2$ solution) under red LED illumination. (b) $\mathrm{pH}$ sensitivity obtained with a spectrophotometer in terms of the reflected intensity of red peaks $(630 \mathrm{~nm})$.

is required. Recent advance of microelectronic technology enables the performance of CMOS image sensors (i.e., intensity resolution, spatial resolution, and signal-to-noise ratio) to be comparable to that of CCD imagers. Therefore, it is expected that the functionality can be largely expanded even with cost-effective CMOS sensors.

The reported application does not require any high spatial resolution with large pixel number since only the average intensity over a single image (only $16 \times 16$ pixels) is of interest. If it is equipped with an imager with higher spatial resolution, however, the mouse configuration has an additional potential merit for microscopic observation, possibly for simple microfluidic cytometry or similar applications.

In this work, the extra instrumentation including the Arduino microcontroller and associated coding was necessary to bypass the sensor signal and visualize the output of image sensor separately. In fact, all the information is to be internally processed within the original mouse circuit board, although not available to the end-users, to conduct the original function of displaying the cursor movement in the computer monitor. This implies that the entire mouse system is readily available for expanded functionalities such as the proposed optochemical quantification. Moreover, the need of additional optical filters in this report can be largely circumvented by adopting color imagers with on-chip RGB Bayer filters. This will extend the functionality of mouse beyond its originally intended use including chemical measurements without significant extra cost.

Currently many optical technologies are being introduced for high-performance assays with ultrahigh sensitivity and wide linear dynamic range toward low-cost medical diagnosis and environmental surveillance with mobile settings [5]. These include nanoparticles, quantum dots, and plasmons, based on lab-on-chip or similar point-of-care (POC) platforms, which are highly feasible to be implemented with the proposed optical mouse platform. Many aforementioned advanced assays require monochromatic and collimated laser light sources. Special-purpose mouse, including the gaming mouse for precise navigation, is equipped with multiple laser diodes (VIS-IR range) and advanced imagers with highly quantized digital output and large number of pixels. Undoubtedly, such high-performance mouse will exhibit a superior capability for the proposed applications.

The preinstalled optical arrangement of mouse is readily available for the general purpose microscopic observation with minimal effort. In many instances of imaging, there are significant problems associated with motion artifacts due to the alignment of instrument. The ability of the optical mouse to detect inadvertent movement can be harnessed in tandem. Therefore, this approach is considered as one viable option to expedite the progress developing low-cost, easily accessible optochemical detection systems with short development time and easier standardization as compared to other popular approaches (e.g., cellphones) [5].

\section{Conclusion}

This work demonstrated for the first time that the optical sensing platform utilizing optical mouse, the easily accessible optoelectronic computer peripheral, can be used for chemical quantification. Particularly, we took advantage of the optical configuration of the optical mouse, composed of imaging device, solid-state light source, and microoptics, to be utilized as the optical sensing platform for oxygen and $\mathrm{pH}$ determination. First, imaging of emission intensity from oxygen sensor patches was conducted with an optical mouse to determine the oxygen level. Secondly, it was used to analyze reflected colors from $\mathrm{pH}$ test strip responding to different $\mathrm{pH}$ values. The black-and-white image sensor inside the optical mouse exhibited good dynamic range and sensitivity for differentiating the oxygen and $\mathrm{pH}$ levels under LED illumination.

Therefore, the optical mouse platform is well suited for optochemical assays to capture chemical images and transmit pixel data functioning as a ubiquitous analytic 

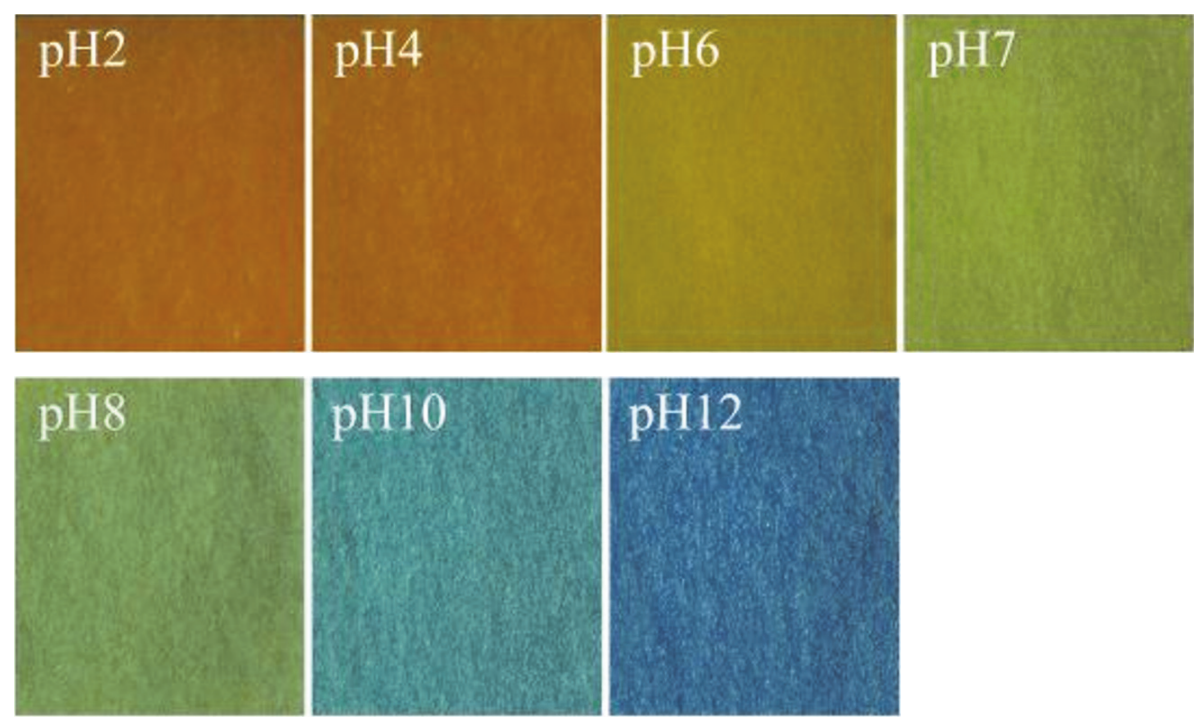

(a)

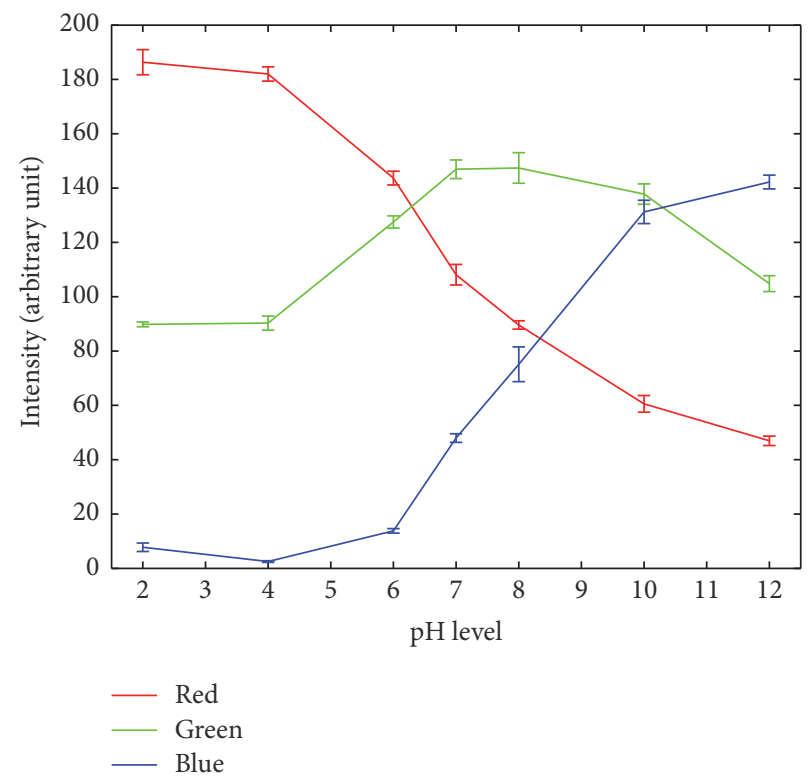

(b)

FIGURE 6: pH analysis with a color camera. (a) Cropped color images of pH test strips obtained with color camera; (b) pH sensitivity obtained with a color camera in terms of the intensities of three primary colors (RGB) $(n=3)$.

platform with network connectivity for quantitative chemical measurements. This approach offers potentially a convenient, affordable, and reliable alternative to traditional instruments for quantifying chemical levels. A possibility is widely open to extend this approach to a variety of advanced optochemical sensing modalities adopting different optical agents and techniques with superior light sources and image sensors.

\section{Conflicts of Interest}

The authors declare that there are no conflicts of interest regarding the publication of this paper.

\section{Acknowledgments}

This work was supported by the IEEE Instrumentation \& Measurement Society (IMS) Graduate Fellowship Award, Center for Biomedical Science and Engineering (CBSE), and Materials Research Center (MRC) at Missouri University of Science and Technology, Rolla, MO.

\section{References}

[1] T. W. Ng, "The optical mouse as a two-dimensional displacement sensor," Sensors and Actuators, A: Physical, vol. 107, no. 1, pp. 21-25, 2003. 
[2] J. Palacin, I. Valgañon, and R. Pernia, "The optical mouse for indoor mobile robot odometry measurement," Sensors and Actuators A: Physical, vol. 126, no. 1, pp. 141-147, 2006.

[3] M. K. Chmarra, N. H. Bakker, C. A. Grimbergen, and J. Dankelman, "TrEndo, a device for tracking minimally invasive surgical instruments in training setups," Sensors and Actuators, A: Physical, vol. 126, no. 2, pp. 328-334, 2006.

[4] U. Minoni and A. Signorini, "Low-cost optical motion sensors: an experimental characterization," Sensors and Actuators A: Physical, vol. 128, no. 2, pp. 402-408, 2006.

[5] A. Rasooly and K. E. Herold, Mobile Health Technologies, Methods and Protocols, Humana Press, New York, NY, USA, 2015.

[6] N. López-Ruiz, A. Martínez-Olmos, I. M. Pérez De VargasSansalvador et al., "Determination of $\mathrm{O}_{2}$ using colour sensing from image processing with mobile devices," Sensors and Actuators, B: Chemical, vol. 171-172, pp. 938-945, 2012.

[7] L. Shen, J. A. Hagen, and I. Papautsky, "Point-of-care colorimetric detection with a smartphone," Lab on a Chip, vol. 12, no. 21, pp. 4240-4243, 2012.

[8] A. Fakki, S. Ahmed, J. Park, and C-S. Kim, "Optical mouse as $\mathrm{pH}$ analyzer," in Proceedings of the 2015 IEEE Sensors, Busan, South Korea, 2015.

[9] X.-D. Wang and O. S. Wolfbeis, "Optical methods for sensing and imaging oxygen: materials, spectroscopies and applications," Chemical Society Reviews, vol. 43, no. 10, pp. 3666-3761, 2014.

[10] S. Tucker, R. Robinson, C. Keane et al., "Colorimetric determination of $\mathrm{pH}$," Journal of Chemical Education, vol. 66, no. 9, pp. 769-771, 1989.

[11] Arduino, 2015, https://www.arduino.cc/.

[12] ADNS 2051, 2015, http://www.alldatasheet.com/datasheet-pdf/ pdf/102950/HP/ADNS-2051.html.

[13] J. Park, W. Hong, and C.-S. Kim, "Color intensity method for hydrogel oxygen sensor array," IEEE Sensors Journal, vol. 10, no. 12, pp. 1855-1862, 2010.

[14] J. Park and C.-S. Kim, "A simple oxygen sensor imaging method with white light-emitting diode and color chargecoupled device camera," Sensor Letters, vol. 9, no. 1, pp. 118-123, 2011.

[15] S. Park, S. G. Achanta, J. Yang, and C.-S. Kim, "Intensity-based oxygen imaging with a display screen and a color camera," Sensors and Actuators, B: Chemical, vol. 164, no. 1, pp. 101-108, 2012. 


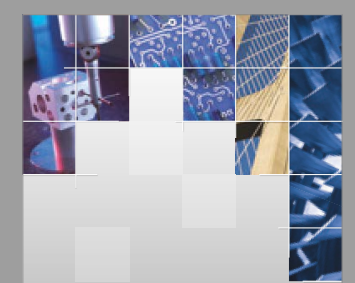

\section{Enfincering}
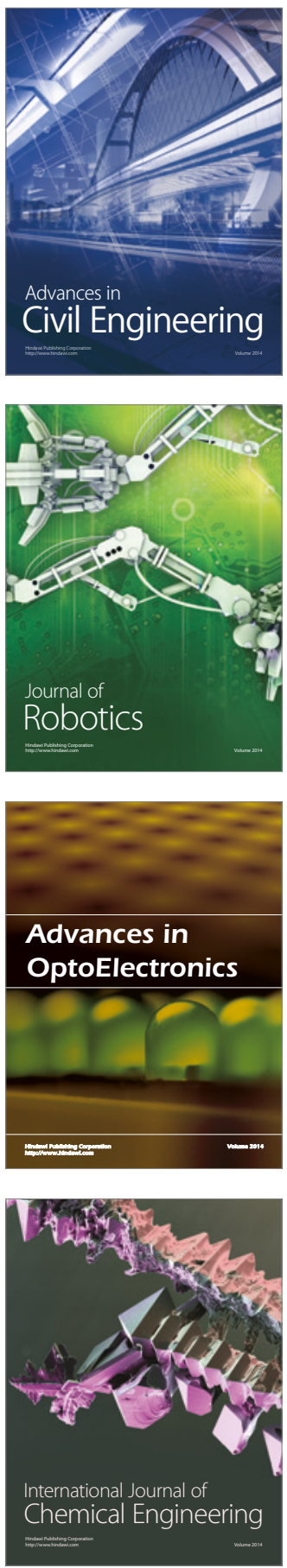

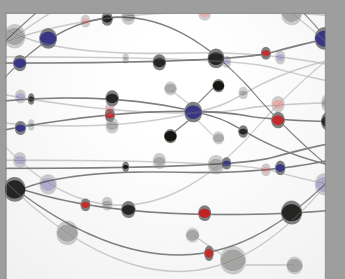

The Scientific World Journal

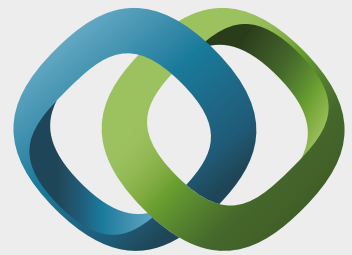

\section{Hindawi}

Submit your manuscripts at

https://www.hindawi.com
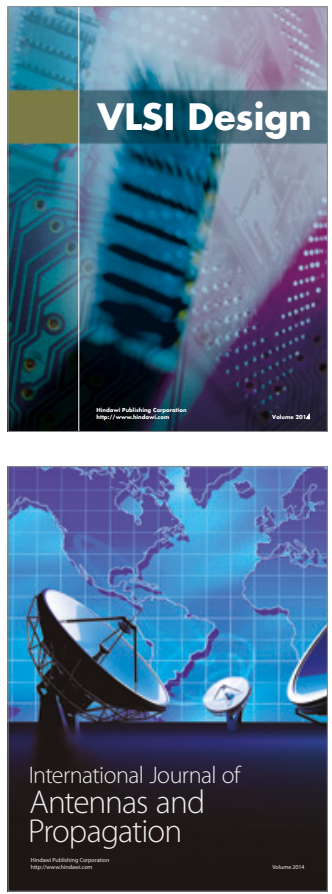

\section{Rotating}

Machinery
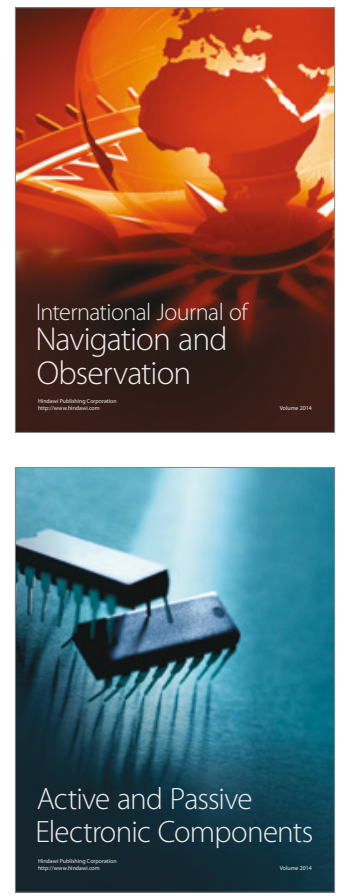
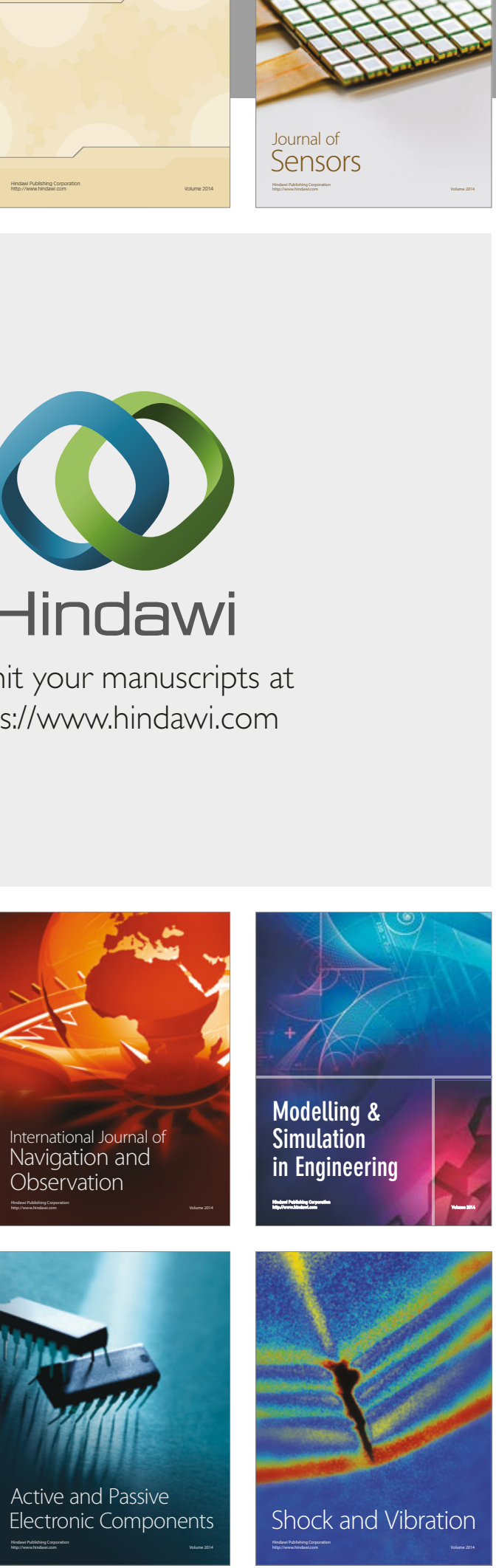
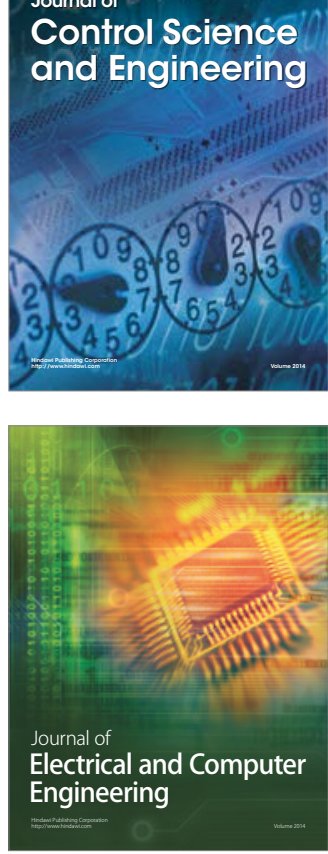

Distributed

Journal of

Control Science

and Engineering
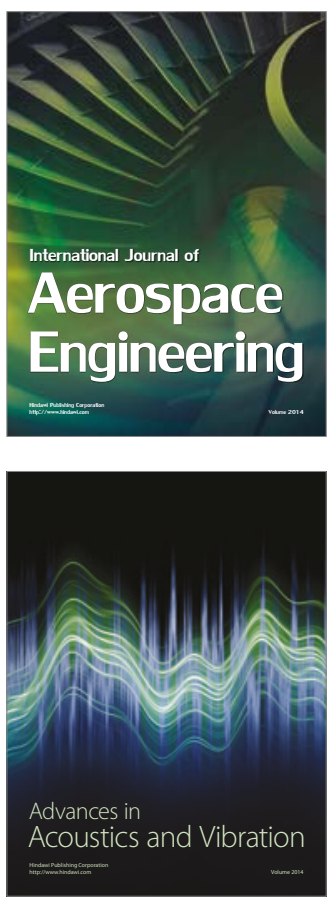

Sensor Networks 